\title{
Biologia floral de Vochysia cinnamomea Pohl (Vochysiaceae) em cerrados do Triângulo Mineiro, MG
}

\author{
MIRLEY LUCIENE SANTOS ${ }^{1}$, ALINE DE PAULA AFONSO $^{1} \mathrm{e}$ \\ PAULOEUGÊNIOOLIVEIRA ${ }^{2}$
}

(recebido em 30/06/96; aceito em 04/12/96)

\begin{abstract}
Floral biology of Vochysia cinnamomea Pohl (Vochysiaceae) in cerrados of the Triângulo Mineiro, MG). Vochysia cinnamomea is a small tree commom in the cerrado vegetation in southern areas of Central Brazil. The yellow zigomorphic flowers have a gamosepalous calix with a larger spurred lobe. The one-day flowers open during the morning and the only stamen is ejected during the anthesis leaving the pollen on the style, a kind of secondary pollen presentation. The most common visitors are Epicharis bees and other large Anthophoridae wich are the main pollinators, although hummingbirds and diurnal sphingids were also observed. The plant is partially self-compatible though mature selfed fruits were barely over a quarter of the crossed ones, and index of self-incompatibility (ISI) of 0.26 . Self pollen tubes are arrested half way down the style in most of the observed pistils wich indicates a functional self-incompatibility mechanism similar to the observed in other species of Vochysia. Pollination and breeding systems features of $V$. cinnamomea are basically similar to the observed for other Vochysia species studied in Brazil, although most of them are more clearly self-incompatible. The partial self-compatibility found here may help to explain the occurrence of totally self-compatible species studied in Costa Rica.
\end{abstract}

RESUMO - (Biologia floral de Vochysia cinnamomea Pohl (Vochysiaceae) em cerrados do Triângulo Mineiro, MG). Vochysia cinnamomea é uma arvoreta de cerrado comum na região do triângulo mineiro. Apresenta flores zigomorfas com pétalas amarelas e cálice gamossépalo com um dos lobos maior e calcarado. As flores abrem pela manhã e duram apenas um dia. Apresentam um estame que se desprende durante a antese, depositando o pólen no estilete, o que representa um tipo de apresentação secundária de pólen. As flores são visitadas principalmente por abelhas do gênero Epicharis e outras Anthophoridae, que são os polinizadores primários, mas beija-flores e esfingídeos diurnos também foram observados. A planta é parcialmente autocompatível, sendo os frutos formados por autopolinização pouco mais que um quarto dos formados por polinizações cruzadas, um índice de auto-incompatibilidade (ISI) de 0,26. Tubos polínicos de autopolinização cessam o crescimento no meio do estilete na maioria dos pistilos, indicando um mecanismo de incompatibilidade semelhante ao de outras espécies de Vochysia. Os sistemas de polinização e reprodução de V. cinnamomea são, de maneira geral, similares aos de outras espécies brasileiras de Vochysia, apesar da maioria ser claramente autoincompatível. A autocompatibilidade parcial na espécie estudada pode explicar a existência de espécies de Vochysia totalmente compatíveis encontradas em Costa Rica.

Key words - Pollination, secondary pollen presentation, large bees, savanna, Vochysiaceae.

\section{Introdução}

A família Vochysiaceae abrange seis gêneros e cerca de 200 espécies tropicais, sem representantes nas regiões temperadas (Stafleu 1948, 1952, 1953). O núcleo de distribuição de suas espécies encontrase na região Guiano-Amazônica e no Planalto Central Brasileiro, de onde se irradiam para as demais regiões (Barroso et al. 1984). Qualea e Vochysia são os maiores gêneros e apesar de serem basicamente florestais apresentam espécies muito comuns e bem distribuídas nos cerrados. O gênero Vochysia tem ampla distribuição nestas áreas, com cerca de 17 espécies citadas para esta vegetação (Stafleu 1948).

1. Pós-graduação em Botânica, Universidade de Brasília,

2. Departamento de Biociências, Universidade Federal de Uberlândia, Caixa Postal 593, 38400-902 Uberlândia, MG, Brasil.
Os estudos sobre biologia reprodutiva das Vochysiaceae ainda são limitados em número. Silberbauer-Gottsberger \& Gottsberger (1975) estudaram a polinização de Qualea grandiflora por mariposas e Barbosa (1983) estudou três espécies de Qualea em cerrados de Brasília. O sistema de reprodução de Vochysia ferruginea foi estudado por Bawa et al. (1985) e um apanhado das características reprodutivas de duas espécies da Costa Rica, $V$. ferruginea e $V$. guatemalensis foi feito por Flores (1993). Recentemente, um estudo envolvendo seis espécies de Vochysia foi realizado por Oliveira \& Gibbs (1994) nos cerrados do Brasil Central. Existem, ainda, dados recém-publicados sobre a biologia floral de Salvertia convallariodora (Oliveira 1996).

A biologia floral de Vochysia cinnamomea Pohl, muito freqüente nos cerrados do município de Uberlândia, foi estudada no presente trabalho e 
comparada com os dados existentes para outras espécies de Vochysiaceae de cerrado.

\section{Material e métodos}

O estudo foi realizado em uma área de preservação de cerrado, com ca. 250 ha, no Clube Caça e Pesca Itororó, município de Uberlândia, MG. As populações na área foram acompanhadas em 1992, 1993 e 1994, observando-se a época de floração e as características florais das plantas. Flores coletadas, frescas ou fixadas em álcool 70\% e FAA 50\%, foram analisadas sob lupa e desenhadas com auxílio de câmara clara. A antese foi seguida em diferentes indivíduos e o volume de néctar foi medido utilizando-se micropipetas de volume definido (Blaubrand-IntraMark ${ }^{\circledR}$ ) para extração. Um refratômetro de mão foi usado para estimar a concentração, em "equivalentes de sacarose" (sensu Inouye et al. 1980).

Os visitantes foram periodicamente observados em suas atividades nas flores e coletados para posterior identificação. A identificação dos visitantes até ao nível de família foi feita por comparação com coleções da Universidade Federal de Uberlândia e, posteriormente, este material foi enviado para identificação por especialistas.

O sistema de reprodução foi estudado em flores previamente isoladas por sacos de tecido fino de organza de nylon. Foram efetuados tratamentos de polinização controlada (adaptado de Bawa 1974) utilizando-se sete árvores distantes mais de $50 \mathrm{~m}$ entre si. Polinizações cruzadas e autopolinizações manuais foram efetuadas em todas plantas, sendo as flores marcadas no pedicelo com cola plástica colorida (cola atóxica de PVA). Outras flores foram marcadas para verificar a ocorrência de polinização em condições naturais. Os frutos foram acompanhados até 30 dias após o início do desenvolvimento. Alguns frutos provenientes de autopolinização foram observados durante a maturação.

Algumas flores nas quais foram feitas autopolinizações e polinizações cruzadas, foram fixadas em FAA 50\% após 24,48 e 72 horas, para observação dos pistilos. Estes foram tratados e corados com azul de anilina, para o estudo do crescimento de tubos polínicos sob microscopia de fluorescência (Martin 1959, modificado como em Proença \& Gibbs 1994).

\section{Resultados}

Vochysia cinnamomea é um arbusto ou arvoreta de até $6 \mathrm{~m}$ de altura ocorrendo em cerrados abertos na região de Uberlândia. O período de floração estendeu-se de março a abril nos três anos do estudo. A inflorescência é longa, atingindo até $40 \mathrm{~cm}$ de comprimento e possui muitos cicinos com dois ou três botões dispostos ao longo de um eixo não ramificado. O número de botões e o tamanho da inflorescência variou entre plantas e dentro de uma mesma planta. As flores são zigomorfas e o cálice é gamossépalo, com um dos lobos muito maior e calcarado (figura 1). A corola é composta por três pétalas oblongas, sendo a central oblonga-obovada e as laterais mais estreitas, ligeiramente recurvadas (figura 2), de cor amarela e vistosas. A pétala central é tão longa quanto o estame. O ovário é súpero e piloso; acima do ovário encontra-se o acesso ao cálcar, onde é produzido o néctar (figura 3). As flores apresentam odor discreto e adocicado.

A antese é diurna e as flores estão totalmente abertas por volta das $10 \mathrm{~h}$ da manhã. A abertura da flor envolve um movimento do lobo maior do cálice que se curva lentamente para trás expondo as pétalas. Durante este processo o estame é submetido a uma certa tensão e, comumente, o filete rompe-se na base. A seguir a antera cai, ficando o pólen depositado sobre a superfície externa do estilete (apresentação secundária de pólen, sensu Yeo 1993). O estigma, na extremidade do estilete e um pouco acima das tecas da antera quando no botão, não é contaminado por este pólen e está receptivo desde o momento da abertura da flor.

O néctar é secretado no lúmen do cálcar. Durante a manhã da antese, um volume médio de $6,7 \mu \mathrm{l}(\mathrm{n}=$ $10 \pm 0,75$ ) foi produzido em flores ensacadas, com uma concentração de equivalentes de sacarose de $36,5 \%( \pm 2,0)$. O volume total produzido e acumulado durante o período de duração de flores isoladas chegou a $35 \mu \mathrm{l}$, mantendo níveis semelhantes de concentração. Os polinizadores visitam as flores à procura do néctar, produzido ativamente no primeiro e segundo dia. No segundo dia, as flores perdem as pétalas ou estas murcham. O estigma se desprende no terceiro ou quarto dia e o cálice murcha lentamente. Na medida em que o ovário começa a se desenvolver o cálice sofre abscisão e se desprende da flor, processo que pode levar mais de uma semana.

Os visitantes das flores estão apresentados na tabela 1. Os visitantes principais e mais comuns foram abelhas grandes, do gênero Epicharis. Estas abelhas sobrevoavam as plantas em grupos da ordem de uma dezena e em surtos periódicos. As abelhas pousam frontalmente nas flores e se agarram às pétalas ou aos lobos do cálice. A probóscide longa é introduzida no cálcar para a retirada do néctar. A abelha pode permanecer por cerca de cinco segundos na flor. Durante a visita, o estilete comumente está posicionado de um lado ou do outro da abelha, de modo que a superfície estigmática não contata o corpo do visitante (figura 4). O contato com o estigma dá-se durante o pouso ou quando a abelha levanta vôo. 


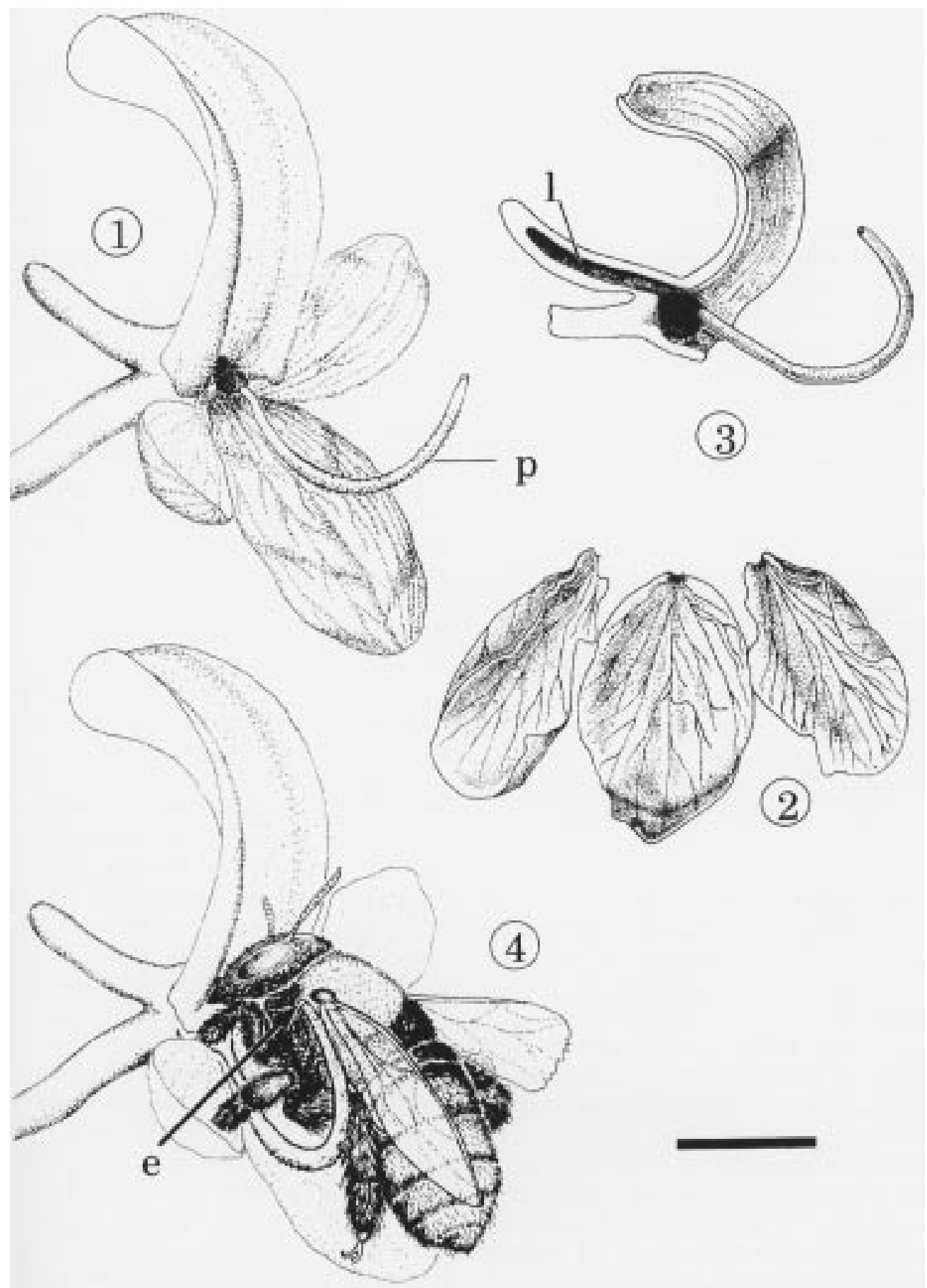

Figuras 1-4. Detalhes da flor de V. cinnanomea. 1. Vista geral da flor com pétalas expandidas e sem o estame que cai durante a abertura, depositando o pólen (p) principalmente na superfície inferior do estilete; 2. Pétalas retiradas de uma flor recém aberta, sendo a pétala central um pouco maior e mais larga que as demais; 3. Corte longitudinal mediano da flor mostrando a posição do ovário piloso e o lúmen (1) do cálice onde é produzido e acumulado o néctar; 4. Epicharis sp. visitando flor de V. cinnamomea. Note a posição do estilete em relação ao corpo da abelha. A superfície estigmática é pequena e situa-se no ápice do estilete (seta). Pousada, a abelha introduz a probóscide acima do ovário procurando o acesso ao néctar no lúmen do cálcar (Ilustração baseada em fotografia). Escala $=1 \mathrm{~cm}$. 
Tabela 1. Visitantes registrados nas inflorescências de $V$. cinnamomea no cerrado estudado.

\begin{tabular}{l}
\hline Insetos \\
\hline Hymenoptera \\
Anthophoridae \\
Acanthopus superba Schrottky \\
Centris fuscata Lepeletier \\
Centris spp. \\
Epicharis analis (Lepeletier) \\
Epicharis melanoxantha Moure \\
Epicharis rustica (Olivier) \\
Epicharis schrottkyi (Friese) \\
Thygather analis (Lepeletier) \\
Apidae \\
Bombus morio (Swerderus) \\
Trigona spinipes (Fabricius) \\
Oxaeidae \\
Oxaea flavescens (Klug.) \\
Lepidoptera \\
Hesperiidae \\
Urbanus sp \\
\hline Apodiformes \\
Trochilidae \\
Amazilia sp* \\
\hline outras duas espécies de beija-flores foram observadas sem \\
\hline
\end{tabular}

Outros visitantes, como os beija-flores e as borboletas, exibiam comportamento oportunístico, coletando o néctar à distância, de modo que raramente contactavam o pólen ou a superfície estigmática.

Os resultados obtidos com os tratamentos de polinização controlada estão representados na tabela 2 e indicam que esta espécie é parcialmente autocompatível, com um índice de autoincompatibilidade (ISI sensu Bullock 1985) de 0,26. Os frutos de autopolinização desenvolveram-se até a maturação. Não foi verificada a viabilidade das sementes resultantes dos tratamentos, mas nenhuma diferença morfológica foi observada.

A análise sob microscopia de fluorescência mostrou que houve crescimento de tubos polínicos em ambos os tratamentos. Para as polinizações cruzadas, os tubos polínicos foram observados alcançando o ovário e penetrando os óvulos 24 horas
Tabela 2. Resultados das polinizações controladas efetuadas em flores de Vochysia cinnamomea, e o índice de autoincompatibilidade obtido a partir destes resultados.

\begin{tabular}{lccc}
\hline Tratamentos & Flores(n) & Frutos(n) & Sucesso(\%) \\
\hline Polinização cruzada & 91 & 25 & 27,5 \\
Auto-polinização & 84 & 6 & 7,1 \\
Condições naturais & 94 & 11 & 11,7 \\
(controle) & & \\
\hline ISI* = 0,26 & & \\
*Índice de autoincompatibilidade (\% de frutos autopolini- \\
zados/\% de frutos de polinização cruzada). 0,25 seria o limite \\
para plantas autoincompatíveis (Bullock 1985).
\end{tabular}

após a polinização ( $\mathrm{n}=10$ pistilos). Na maioria dos pistilos autopolinizados, o crescimento foi interrompido no estilete, e os tubos polínicos não alcançaram o ovário. Entretanto, foram observados dois casos de autopolinizações nas quais os tubos polínicos alcançaram o ovário e penetraram os óvulos ( $\mathrm{n}=9$ pistilos $)$.

\section{Discussão}

O gênero Vochysia apresenta notável semelhança quanto à morfologia floral e as espécies parecem estar adaptadas aos mesmos tipos de polinizadores (Oliveira \& Gibbs 1994), que são principalmente abelhas grandes (sensu Frankie et al. 1983). Nesse sentido, V. cinnamomea se enquadra bem nas características observadas para outras espécies de Vochysia (Oliveira \& Gibbs 1994). O tipo de mecanismo de abertura da flor e apresentação secundária de pólen é semelhante ao de outras espécies do gênero. Esta apresentação secundária do pólen pode ser classificada como do tipo "pseudoestame" (Yeo 1993), funcionando o estilete como a estrutura que deposita o pólen no corpo dos polinizadores. Um mecanismo semelhante de apresentação secundária de pólen ocorre em Salvertia convallariodora, onde a antera afasta-se do centro da flor durante a antese e parece não ser importante na polinização propriamente dita (Oliveira 1996). Entretanto, a ejeção da antera durante a antese parece ser característica das espécies de Vochysia.

Outro aspecto interessante, observado também para outras espécies de Vochysia, é que apesar de ser polinizada por abelhas grandes $V$. cinnamomea é visitada por beija-flores e borboletas, que podem 
funcionar como polinizadores secundários. O volume e a concentração de néctar produzido são semelhantes aos de outras espécies de Vochysia (Oliveira \& Gibbs 1994) e de outras plantas polinizadas por abelhas (Barbosa 1983, Baker \& Baker 1983), mas atraem também estes visitantes e possíveis polinizadores secundários.

Vochysia cinnamomea tem inflorescência e flor semelhante a $V$. rufa (Oliveira \& Gibbs 1994) que ocorre também em Uberlândia. Estas espécies são muito semelhantes morfologicamente, sendo por vezes de difícil distinção taxonômica. Entretanto, segundo Stafleu (1948) e K. Yamamoto (comunicação pessoal) podem ser consideradas duas espécies diferentes. Em ambas as espécies, o tamanho da flor parece ser compatível com a polinização por abelhas solitárias grandes.

Os resultados obtidos nos tratamentos de polinizações controladas em Vochysia cinnamomea, mostraram que a planta é parcialmente autocompatível, ou seja, o ISI que é de 0,26, está no limite considerado para plantas autoincompatíveis (Bullock 1985). Estes resultados para V. cinnamomea são semelhantes aos obtidos por Oliveira \& Gibbs (1994), para V. elliptica. No entanto, um outro estudo com outros indivíduos de $V$. cinnamomea na mesma área, revela uma porcentagem de frutos formados por autopolinizações muito baixa $(1,28 \%, \mathrm{n}=78)$ e o ISI calculado a partir desse estudo indica plantas fortemente autoincompatíveis (P. Borges, dados não publicados). Diferenças na intensidade da autoincompatibilidade, observadas em outros estudos de plantas tropicais (Bawa 1979 e N. Ramirez, comunicação pessoal), talvez expliquem os resultados distintos obtidos. Nas espécies de Vochysia apenas V. ferruginea, em Costa Rica (Bawa et al. 1985), parece ser caracteristicamente autocompatível. Esta espécie apresentou ISI em torno de 0,95 , isto é, número semelhante de frutos formados por autopolinização e polinização cruzada. Também em Costa Rica, V. guatemalensis é citada como autocompatível (Flores 1993), mas nenhum detalhe sobre esta caracterização é fornecido. A maior parte das Vochysiaceae estudadas até o momento é autoincompatível (Barbosa 1983, Oliveira \& Gibbs 1994), embora Salvertia convallariodora, espécie de um gênero monotípico e com características relativamente primitivas entre as Vochysiaceae, também apresente autocompatibilidade (Oliveira 1996).

O padrão de crescimento dos tubos polínicos na espécie estudada também é semelhante ao de outras espécies de Vochysia. Oliveira \& Gibbs (1994) estudaram seis espécies em Brasília, todas apresentando certo grau de autoincompatibilidade. Nestas plantas o crescimento de tubos polínicos provenientes de autopolinização cessa no segundo terço do estilete e formam-se deposições irregulares de calose. Nos pistilos de V. cinnamomea as deposições de calose não foram tão evidentes, mas os tubos em muitas autopolinizações tiveram atividade semelhante, cessando de crescer na primeira metade do estilete. Este padrão, que pode ser considerado um tipo "clássico" de reação de incompatibilidade, é associado a sistemas genéticos de incompatibilidade gametofíticos (Nettancourt 1977, Gibbs 1990). Esta reação é diferente da observada em Qualea spp. (Oliveira 1991), nas quais os tubos polínicos, tanto de autopolinização quanto polinização cruzada, crescem até o ovário e os fenômenos de autoincompatibilidade têm ação tardia (sensu Seavey \& Bawa 1986).

Este estudo confirma a relativa homogeneidade na biologia floral de espécies de Vochysia, envolvendo inclusive mecanismos de incompatibilidade semelhantes. A autocompatibilidade parcial na espécie estudada sugere que estes sistemas podem apresentar relativa flexibilidade nas espécies de Vochysia e explicam a existência de espécies totalmente autocompatíveis.

Agradecimentos - Ao Depto. de Biociências e à Universidade Federal de Uberlândia pelo apoio durante a realização do trabalho. Ao Clube Caça e Pesca Itororó pela permissão para trabalhar em sua área de preservação. Ao Dr. Anthony Raw pela identificação das abelhas. À Simone Carolina S. Silva pela finalização das pranchas de morfologia floral. Ao CNPq pelas bolsas de Iniciação Científica e Pesquisa do 2 e 3autores (Proc. № 502671/91-2). A 1ㄹ autora teve bolsa do PET-Biologia (CAPES-UFU) durante a realização do trabalho.

\section{Referências bibliográficas}

Baker, H.G. \& Baker, I. 1983. Floral nectar sugar constituents in relation to pollinator type. In Handbook of experimental pollination biology (C.E. Jones \& R.J. Little, eds.). Van Nostrand Reinhold Co., New York, p. 117-141.

Barbosa, A.A. 1983. Aspectos da ecologia reprodutiva de três espécies de Qualea (Vochysiaceae) num cerrado de Brasília - D.F. Dissertação de mestrado, Universidade de Brasília, Brasília. 
Barroso, G.M., Peixoto, A.L., Costa, C.G., Ichaso, C.F., Guimarães, E.F. \& Lima, H.C. 1984. Sistemática de angioespermas do Brasil. v. 2. Imprensa Universitária, Viçosa.

Bawa, K.S. 1974. Breeding systems of tree species of a lowland tropical community. Evolution 28:85-92.

Bawa, K.S. 1979. Breeding systems of trees in a tropical wet forest. New Zeal. J. Bot. 17:521-524.

Bawa, K.S., Perry, D.R. \& Beach, J.H. 1985. Reproductive biology of tropical lowland rain forest trees. I. Sexual systems and incompatibility mechanisms. Amer. J. Bot. 72:331-345.

Bullock, S.H. 1985. Breeding systems in the flora of a tropical deciduous forest. Biotropica 17:287-301.

Flores, E.M. 1993. Vochysia guatemalensis y Vochysia ferruginea. Arboles y Semillas del Neotropico (Costa Rica) 2:1-52.

Frankie, G.W., Harber, W.A., Opler, P. A. \& Bawa, K.S. 1983. Characteristics and organization of the large bee pollination system in the Costa Rican dry forest. In Handbook of experimental pollination biology (C.E. Jones \& R.J. Little, eds.). Van Nostrand Reinhold Co., New York. p.411-447.

Gibbs, P.E. 1990. Self-incompatibility in flowering plants: a neotropical perspective. Revta brasil. Bot. 13:125-136.

Inouye, D.W., Favre, N.D., Lanun, J.A., Levine, D.M., Meyers, J. B., Roberts, M.S., Tsao, F.C. \& Wang, Y.Y. 1980. The effect of non-sugar nectar constituents on estimates of nectar energy content. Ecology 61:992-995.

Martin, F.N. 1959. Staining and observing pollen tubes in the style by means of fluorescence. Stain Tech. 34:125-128.
Nettancourt, D. de 1977. Incompatibility in angioesperms. Springer-Verlag, Berlin.

Oliveira, P.E. 1991. The pollination and reproductive biology of a cerrado woody community in Brazil. PhD Thesis, University of St. Andrews, Saint Andrews.

Oliveira, P.E. 1996. Polinização e sistema de reprodução de Salvertia convallariodora (Vochysiaceae): uma espécie de cerrado polinizada por mariposas (LepidopteraSphingidae). Revta brasil. Bot. 19:49-53.

Oliveira, P.E. \& Gibbs, P.E. 1994. Pollination and breeding systems of some Vochysia species (PolygalalesVochysiaceae) in Central Brazil. J. Trop. Ecol. 10:509-522.

Proença, C.E.B. \& Gibbs, P.E. 1994. Reproductive biology of eight sympatric Myrtaceae from Central Brazil. New Phytol. 126:343-354.

Seavey, S.R. \& Bawa, K.S. 1986. Late-acting self-incompatibility in angiosperms. Bot. Rev. 52:195-219.

Silberbauer-Gottsberger, I. \& Gottsberger, G. 1975. Uber sphingophile Angiospermen Brasiliens. Pl.Syst.Evol. 123:157-184.

Stafleu, F.A. 1948. A monography of the Vochysiaceae. I. Salvertia and Vochysia. Meded. Bot. Mus. Utr. 95:397540.

Stafleu, F.A. 1952. A monography of the Vochysiaceae. II. Callisthene. Acta Bot. Neerl. 1:222-242.

Stafleu, F.A. 1953. A monography of the Vochysiaceae. III. Qualea. Acta Bot. Neerl. 2:144-217.

Yeo, P.F. 1993. Secondary pollen presentation: form, function and evolution. Springer-Verlag, Wien. 